\title{
Comparison of cell-mediated immunity due to Leishmania infantum promastigotes and axenic amastigotes
}

\author{
Najafi-Dastanaei $\mathbf{A}^{\mathbf{1}}$, Ajdary $\mathbf{S}^{\mathbf{1}}$, Khaze $\mathbf{V}^{\mathbf{1}}$, Darabi $\mathbf{H}^{\mathbf{1}}$, Alimohammadian $\mathrm{MH}^{1 *}$ \\ ${ }^{1}$ Pasteur Institute of Iran, Department of Immunology, 69 Pasteur Avenue, Tehran 13169-43551, Iran.
}

\begin{abstract}
Introduction: Leishmania infantum is the causative agent of visceral leishmaniasis (VL). Cell-mediated immunity (CMI) is required to control leishmaniases. Therefore, simple tests that can evaluate the cellular immunity of the target populations can help to understand the immune status of the human subjects, their immunity to the re-infection and the evaluation of the effectiveness of the potential vaccines. Here, we compared antigens based on single clones of $L$. infantum promastigotes and axenic amastigotes by in vitro and in vivo tests. Methods: Using serial dilutions, $L$. infantum promastigotes were selected as single clones (PSC) or were grown under axenic conditions with succinate-tris to prepare amastigote-like single clones (ASC). Antigens prepared from PSC and ASC were then compared with typical Leishmania major and L. infantum amastigotes by SDS-PAGE, Western-blotting and proliferation tests as well as an in vivo delayed-type hypersensitivity test on guinea pigs. Results Both PSC and ASC exhibited a distinctive $\sim 50-\mathrm{kDa}$ band could be detected by Western-blotting. The proliferation tests results indicated that both PSC and ASC could cause higher lymphocyte proliferation compared to typical L. infantum and L. major promastigotes; however the differences were not significant. Moreover, both PSC and ASC had an ability to induce comparable DTH and hence CMI. Conclusion: Similar proliferation or delayed-type hypersensitivity could be caused with antigens based on PSC, ASC or the typical promastigotes and any of these reagents could potentially be used for in vivo detection of CMI in VL epidemiological or vaccine studies.
\end{abstract}

KEYWORDS: Visceral leishmaniasis, Leishmania infantum, skin test; Leishmanin, delayed-type hypersensitivity (DTH) test, lymphocyte proliferation assay.

\section{INTRODUCTION}

Leishmania species are a group of parasites that are transmitted by sandfly vectors and cause diverse clinical manifestations, mostly in tropical and subtropical regions of the world. The infections may present themselves as a spectrum of outcomes from self-healing cutaneous lesions to lethal visceral leishmaniasis (VL; also known as kala-azar). These protozoa have a digenetic life cycle, consisted of an extracellular stage when as promastigotes multiply and develop within the digestive tract of their vectors and an intracellular stage, during which they reside and multiply as amastigotes inside the parasitophorous vacuoles of macrophages of their mammalian hosts $[1,2]$. Incidences of leishmaniases have been reported in 98 countries on 5 continents [3]. Leishmaniases are still a major public health problem worldwide with more than 350 million people at risk, among them; the VL victims are accounted for over 50,000 deaths annually [4].

*Corresponding author: Mohammad Hossein Alimohammadian, Pasteur Institute of Iran, Department of Immunology, 69 Pasteur Avenue, Tehran 13169-43551, Iran.

E-mails: mhalimoham@gmail.com

Tel-Fax: (+9821) 64112840
The genus Leishmania comprises of approximately 35 species while 20 among them are responsible for human diseases. The outcome of the infection depends on the biological and genetic characteristics of both the host and the infecting parasite species [5]. VL is caused by L. infantum and L. donovani in the Old World and L. chagasi in the New World [6]. Approximately, 500,000 new cases of VL occur annually $[7,8]$ among which $90 \%$ are detected in 5 countries, namely India, Nepal, Bangladesh, Sudan and Brazil [3]. When infected female sandflies bite a mammalian host, the metacyclic promastigotes are injected into the skin where they are captured by resident phagocytic cells, such as dermal macrophages, keratinocytes and dendritic cells through a CR3-dependent mechanism and will eventually metamorphose to amastigotes (Mougneau et al., 2011). In its amastigote form, the pathogen such as L. infantum proliferates inside the host macrophages and induces cellmediated immunity (CMI). The overall immune system and in particular, the host's CMI, play important roles in outcome of the clinical state of the disease[9]. Therefore, the evaluation of host CMI can help us to understand the status of the disease in the host. The control of leishmaniasis infection is performed through 
the development of Th1 responses and the production of major cytokines of this type of response, mainly IL-12 and IFN- $\gamma$ [1]. The delayed-type hypersensitivity (DTH) reaction is the major in vivo indicator of CMI. The evaluation of CMI can be performed by in-vitro lymphocyte proliferation assay and by in-vivo Leishmanin skin test (LST; previously known as Montenegro test which reveals a DTH reaction). In fact, LST is the only in vivo test for evaluation of CMI in leishmaniases. LST remains positive (meaning, formation of an induration more than $5 \mathrm{~mm}$ after $0.1 \mathrm{ml}$ intradermal injection of Leishmanin) in cutaneous leishmaniasis (CL) patients for many years even after their recovery [10-12]. The result of LST for patients suffering from VL is usually negative; however after treatment and/or recovery, the majority of the patients exhibit a positive LST response [13, 14]. LST has many applications in leishmania-related epidemiological studies which are required to monitor the frequency, the incidence rate, the scale of endemicity, the transmission risk of the infection [15-18], the evaluation of immunogenicity [19-21], and the evaluation of efficacy of the vaccine candidates [22-25]. In practical terms, LST is used in selection of volunteers for assessment of the vaccines efficacy in leishmaniases studies, in order to separate people who had been previously infected by Leishmania species and those with no encounter with the pathogen. Also, LST can be used in diagnosis of the diseases when the number of parasites is so low that cannot be detected with other methods [22-27].

Since only the amastigote form of Leishmania replicate inside the macrophages of their mammalian hosts and its antigens induce acquired CMI, we attempted to evaluate and compare antigen preparations based on single clones of $L$. infantum promastigotes as well as axenic amastigotes in terms of their induction of CMI both in vitro and in vivo.

\section{MATERIALS and METHODS}

\section{Animals}

Female BALB/c mice (6-8 weeks old) and female guinea pigs (3month-old) were purchased from the animal facility of Production Complex of Pasteur Institute of Iran in Karaj. The approval for the experiments was confirmed by Ethical Committee of Pasteur Institute of Iran.

\section{Preparation of the promastigotes}

\section{L. infantum (MHOM/IR/04/IPI-UN10) or L. major} (MRHO/IR/75/ER) were cultured to a stationary phase at $24^{\circ} \mathrm{C}$ in RPMI 1640 medium (Biosera, France), supplemented with $10 \%$ fetal calf serum (FCS; Gibco, Germany), $100 \mathrm{UI} / \mathrm{ml}$ penicillin and $100 \mu \mathrm{g} / \mathrm{ml}$ streptomycin (henceforth called, complete RPMI). After 6 days, when the number of the parasites reached to $25-30 \times 106 / \mathrm{ml}$ promastigotes, the cultures were centrifuged $(3220 \times \mathrm{g}, 20 \mathrm{~min}$, room temperature $)$ and the promastigotes were washed and counted by Neobar hemocytometer (Germany). The parasites were then diluted in PBS (pH 7.2) to a concentration of $5 \times 106 / \mathrm{ml}$ and kept in liquid nitrogen at $-196^{\circ} \mathrm{C}$.

\section{Preparation of promastigote single clones (PSC)}

A batch of above-mentioned $L$. infantum stock sample was cultured in Novy-MacNeal-Nicolle (NNN) slant at $24^{\circ} \mathrm{C}$ for 4 days. The promastigote-containing solution was then transferred to a $5 \mathrm{x}$ volume of complete RPMI. The culture was incubated at $24^{\circ} \mathrm{C}$ for 2 days and then counted and diluted in complete RPMI to 106 promastigotes $/ \mathrm{ml}$. This preparation was two-fold serially diluted in a 96-well plate containing $100 \mu \mathrm{l}$ complete RPMI inside each well, up to 24 serial dilutions. Following incubation of the plate at $24^{\circ} \mathrm{C}$ for $1 \mathrm{~h}$, the cells were examined by an inverted microscope (Leitz, Germany) to find a single parasite cell, trapped in one well due to the dilution. Henceforth, such cells are referred as single-clones. The content of these selected wells were each transferred to a NNN slant. To enhance the accuracy and reliability of this work, the procedure of selecting the single clones was continued as above for 2 more times ( 3 times in total). The total procedure of obtaining single clones was repeated 4 more times, in order to prepare a total of 5 promastigote single clones (i.e PSC1-PSC5). The selected single clones were grown in complete RPMI as above and then were washed twice and diluted in PBS ( $\mathrm{pH} 7.2)$ to a final concentration of $200 \times 106 / \mathrm{ml}$ and then kept at $-80^{\circ} \mathrm{C}$.

Preparation of axenic amastigote-like single clones (ASC) Five prepared PSCs as above were transferred to complete RPMI, supplemented up to $25 \%$ (v/v) FCS. The cultures were incubated at $35.5^{\circ} \mathrm{C}$ (with $5 \% \mathrm{CO} 2$ ) for $18 \mathrm{~h}$. Following centrifugation $(1200 \times \mathrm{g}, 10 \mathrm{~min}$, room temperature $)$ and resuspension in fresh supplemented medium as above (adjusted to $\mathrm{pH} 5.5$ using $10 \mathrm{mM}$ succinate-tris), the suspension was incubated at $35.5^{\circ} \mathrm{C}(5 \% \mathrm{CO} 2)$ for $120 \mathrm{~h}$. When the promastigotes lost their flagella, the preparation was considered as ASC [28-30]. The culture was then centrifuged $(3220 \times \mathrm{g}, 20$ min, room temperature), washed twice with PBS ( $\mathrm{pH} 7.2)$ and stored as ASC1-ASC5 at concentration of $200 \times 106 / \mathrm{ml}$ at $-80^{\circ} \mathrm{C}$. Preparation of mouse anti-L. infantum polyclonal antibody

To obtain the required antibody (IgG) for the Western blotting, BALB/c mice (6-8 weeks old) were injected in the base tail with killed $L$. infantum suspensions $(20 \times 106 / 50 \mu 1)$ mixed with equal volume of Freund's complete adjuvant (Sigma) as the first immunization. The second and third immunizations were followed as above, except they were performed with Freund's incomplete adjuvant. All injections were administered subcutaneously with 2 -week-intervals. Blood $(0.5-\mathrm{ml}$ in total; pooled) was taken from the mice by retro-orbital bleeding procedure at weeks 4,6 and 8 post-injections and serum was collected after centrifugation (10 min, $1 \times \mathrm{g}$ ).

\section{Protein analyses of the prepared clones by SDS-PAGE and} Western blotting

The protein samples were prepared from a boiled $(5 \mathrm{~min})$ suspension of $200 \times 106$ cells from each group. After subjecting $60-\mu 1$ of each sample to SDS-PAGE (12\%), the gel was stained by Coomassie R-250. For Western blotting, the antigens were transferred from the gels to a nitrocellulose membrane (Sigma, Germany) using an electro-transfer semidry device (Pharmacia LKB, Sweden). The membrane was blocked by $1 \%$ (w/v) BSA in PBS. The transferred immunoblots were detected following 2$\mathrm{h}$ incubation at room-temperature with a 1/100 dilution mouse anti- $L$. infantum polyclonal antibody, followed by $2-\mathrm{h}$ roomtemperature incubation with goat anti-mouse IgG-HRP antibody (1/4000 dilution; Sigma, Germany). The bands were developed by diamino-benzamidine-tetrahydrochloride (DAB; Sigma, Germany).

\section{Lymphocyte proliferation assay}

The cells of inguinal lymph node of the immunized mice were suspended in complete RPMI. The cells were then re-suspended in complete RPMI medium plus $1 \%$ L-glutamine. The suspensions (200 $\mu \mathrm{l} / \mathrm{well}$, equivalent of $4 \times 105$ cells) were incubated in presence of $10 \mu \mathrm{l}$ of each whole-cells lysate of the ASC and the PSC preparations as well as $L$. major and $L$. infantum promastigote antigens. For each antigen, a negative control (without antigen) and a positive control (2 $\mu \mathrm{g}$ 
Concanavalin A; Sigma) were used. The cells were incubated in a humidified atmosphere at $37^{\circ} \mathrm{C}(5 \% \mathrm{CO} 2)$ for 3 days and during the last $18 \mathrm{~h}$, the cultures were pulsed with $20 \mu \mathrm{l}(0.5 \mu$ $\mathrm{Ci} /$ well) $[3 \mathrm{H}]$-thymidine (Amersham, UK) per well. The cells were harvested onto glass fiber filters (Whatman, USA). After adding $2 \mathrm{ml}$ liquid scintillation reagent (Sigma, Germany) to all samples, $[3 \mathrm{H}]$-thymidine incorporation into DNA was determined by a liquid scintillation beta-counter (Wallac 1410 LKB, Sweden), measured as counts per minute (CPM).

Immunization and DTH assay on guinea pigs against $L$. infantum

Two-month-old Guinea pigs were immunized in both hind legs intramuscularly once and intradermally three times with $25 \times 106$ freeze-thawed whole $L$. infantum preparations with 2 -week intervals. The first injection contained complete Freund's adjuvant while the second and third injections contained incomplete Freund's adjuvant and the fourth injection contained no adjuvant. After the 8th week, a $100-\mu 1$ volume of each parasite preparation $(12 \times 106 / \mathrm{ml})$ was injected intradermally into the shaved abdominal areas of the animals with $2-\mathrm{cm}$ distance between each injection. The diameters of the ensued indurations were measured with a ruler after 24,48 and $72 \mathrm{~h}$.

\section{Statistical methods}

The data were expressed as Mean \pm SD. Statistical analysis was performed using Prism software (version 6.0; GraphPad, USA).The Student's t-test and ANOVA were used for comparison of differences between study groups. Differences were considered to be statistically significant at $* \mathrm{P}<0.05$, $* * \mathrm{P}<$ $0.01, * * * \mathrm{P}<0.001$.

\section{RESULTS}

\section{SDS-PAGE analysis of the leishmanial proteins}

The Mw of $L$. infantum promastigotes (PSC1-PSC5) and axenic amastigotes single clones (ASC1-ASC5) in comparison with $L$. major $(\mathrm{Lm})$ and L. infantum $(\mathrm{Li})$ total promastigote proteins as evaluated by SDS-PAGE are depicted in Fig. 1. The most pronounced bands associated with PSC consisted of one band with $\sim 50 \mathrm{kDa} M w$ and 2 bands within the range of 60-66 kDa. Meanwhile, ASC preparations had the same $\sim 50 \mathrm{kDa}$ Mw band and only a single band with $\sim 60 \mathrm{kDa} \mathrm{Mw}$. Moreover, compared to PSC samples, all ASC preparations exhibited a band with $~ 17$ $18 \mathrm{kDa}$ Mw.

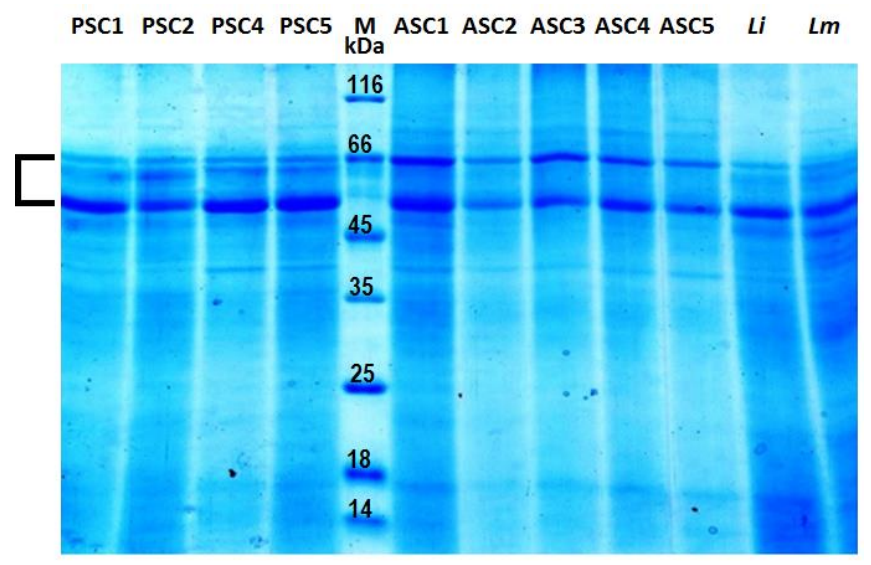

Fig. 1. Comparison of the protein profiles of $L$. infantum PSC and ASC preparations with total promastigote proteins of $L$. major $(\mathrm{Lm})$ and $L$. infantum $(\mathrm{Li})$. $\mathrm{M}$ is $\mathrm{Mw}$ marker.

\section{Western blotting of PSC and ASC}

Antigenic proteins of the prepared PSC and ASC could be detected by Western blotting using a polyclonal serum from mice which had been immunized with $L$. infantum promastigotes. As seen in Fig. 2, a selection of PSC tested and all five ASC antigens were detected as multiple bands, mostly with $\mathrm{Mw}$ of $\sim 50-70 \mathrm{kDa}$.

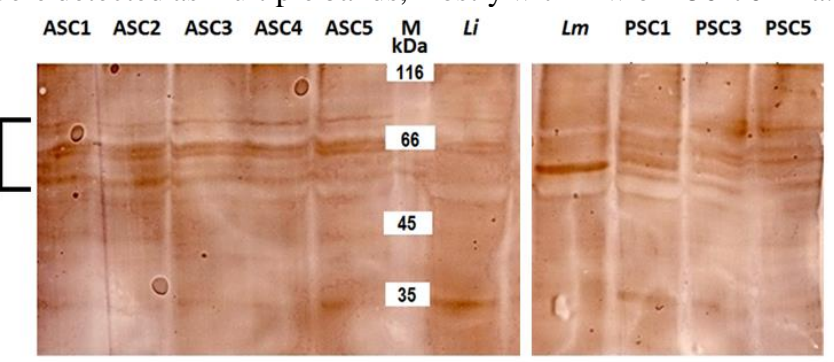

Fig. 2. Western blotting detected ASC and PSC antigens within 50-70 kDa range. ASC, M, Li, Lm, PSC represent axenic amastigote-like single clones, $\mathrm{Mw}$ marker, $L$. infantum and L. major promastigote proteins, respectively.

\section{In-vitro lymphocyte proliferative responses}

Although ASC and PSC could induce higher cell proliferation compared to $L$. major and $L$. infantum total promastigote proteins, as shown in Table 1, the differences in proliferative responses of the lymphocytes were not statistically significant.

Table 1: Mean proliferative responses by ASC and PSC preparations in comparison to $L$. major $(\mathrm{Lm})$ and $L$. infantum ( $\mathrm{Li}$ ) total promastigote proteins and ConA (positive control).

\begin{tabular}{|c|c|c|c|c|c|}
\hline Reagent & $A S C$ & $P S C$ & $L m$ & $L i$ & ConA \\
\hline $\begin{array}{c}\text { Mean CPM } \\
\pm S E M\end{array}$ & $\begin{array}{c}125.6 \\
\pm 6.199\end{array}$ & $\begin{array}{c}123.1 \\
\pm 9.586\end{array}$ & $\begin{array}{c}111.9 \\
\pm 10.87\end{array}$ & $\begin{array}{c}114.4 \\
\pm 7.467\end{array}$ & $\begin{array}{c}6626 \\
\pm 3705\end{array}$ \\
\hline
\end{tabular}

\section{In-vivo DTH responses}

The in vivo results of DTH responses in guinea pigs showed no significant differences between the mean indurations caused by intradermal injection of ASC and PSC, compared to L. infantum and L. major total promastigote proteins after 48 and 72 hours, as shown in Table 2.

Table 2: Means of DTH response of guinea pigs $(\mathrm{n}=3)$, elicited by PSC and ASC, compared to Leishmanin and $L$. infantum $\mathrm{Li}$ ) poly-clone after 24,48 and $72 \mathrm{~h}$.

\begin{tabular}{|c|c|c|c|}
\hline \multirow{2}{*}{ Stimulant } & \multicolumn{3}{|c|}{ Mean diameter of indurations \pm SEM } \\
\cline { 2 - 4 } & $\mathbf{2 4} \mathbf{h}(\mathbf{m m})$ & $\mathbf{4 8} \mathbf{h}(\mathbf{m m})$ & $\mathbf{7 2} \mathbf{h}(\mathbf{m m})$ \\
\hline PSC & $6.56 \pm 0.31$ & $7.27 \pm 0.34$ & $7.77 \pm 0.27$ \\
\hline $\mathbf{A S C}$ & $6.7 \pm 0.24$ & $7.37 \pm 0.43$ & $8.03 \pm 0.45$ \\
\hline $\boldsymbol{L m}$ & $7.25 \pm 0.11$ & $7.67 \pm 0.53$ & $8.41 \pm 0.54$ \\
\hline $\boldsymbol{L i}$ & $8.0 \pm 0.31$ & $8.0 \pm 0.22$ & $8.5 \pm 0.6$ \\
\hline $\begin{array}{c}\text { PBS } \\
\text { (control) }\end{array}$ & 0 & 0 & 0 \\
\hline
\end{tabular}




\section{DISCUSSION}

During leishmaniasis, the host relies on CMI to counteract the intracellular parasite. Therefore, the tests that involve the detection of CMI are considered as valuable tools for evaluation of a person's state of immunity, as well as his/her susceptibility for the re-infection. Moreover, in vaccine studies against leishmaniases, the evaluations of CMI of different study groups are required. One major determinant of CMI toward infections by Leishmania spp. is DTH [31] which for its monitoring, LST is the only in-vivo available test [32]. In patients whose infections progress toward VL, the development of their Th1 responses against the infection is suppressed and their mononuclear blood cells cannot adequately produce IFN- $\gamma$ and IL-12 against the leishmanial antigens. Moreover in such patients, DTH skin tests cannot detect the infection. Although anti-leishmanial antibodies in VL patients are produced in high titers, such antibodies are not protective and are a consequence of polyclonal B cells development [2].

Similar to Leishman in which acts as a reagent to detect the previous exposures to $L$. major antigens, we believe an $L$. infantum-based reagent could be beneficial in endemic areas of VL for the same purpose. Therefore, to choose the best approach, we attempted to compare PSC and ASC as reagents to assess CMI, as well as typical L. infantum and L. major promastigote preparations. The obtained SDS-PAGE results indicated a difference between the prepared ASC and the other tested polypeptides. Unlike other tested samples, here the ASC samples lacked the second pronounced band located at $\sim 60 \mathrm{kDa}$ region and had a more pronounced band with $\sim 17-18 \mathrm{kDa} \mathrm{Mw}$. This indicated that different proteins are expressed under conditions of axenic amastigote preparation.

Our results pertaining to the cell proliferation showed that the lymphocytes stimulated with $L$. infantum ASC (CPM 126) and PSC (CPM 123) were more proliferated compared to either $L$. major (CPM 112) or L. infantum (CPM 114) promastigotes; however these differences were not significant. The observed proliferations caused by L. infantum ASC or PSC were probably due to the existence of more specific immunogens in these preparations. The almost similar and acceptable results of $L$. infantum ASC and PSC in comparison with L. major responses indicate that these preparations are capable of $\mathrm{T}$ cell proliferation in-vitro. Furthermore, our results were in accordance with another study in which lymphocytes from the healed CL patients were confronted with leishmanial antigens of the Old World, namely L. infantum, L. tropica, L. major and L. donovani (Vahid, 1998).

The immunogenicity and efficacy of ASC to induce CMI were compared with L. major in-vivo, using immunized guinea pigs. These experiments showed that ASC can induce DTH, similar to PSC of L. infantum, as well as L. infantum and L. major typical promastigotes, after the intradermal injection. Our results were comparable with earlier results obtained from a $56 \mathrm{kDa}$ L. major antigen in which SLA and culture supernatants could induce DTH [12]. Here, most obtained antigenic bands were $\sim 50 \mathrm{kDa}$ which could have stimulated the immune system of the guinea pigs, confirmed by Western blotting. On the other hand, the observed differences could be due to different potentials of the two different strains of L. major and L. infantum.

In conclusion, our results based on comparison of the single clones by lymphocyte proliferation and skin assays indicated that L. infantum clones could significantly induce DTH in guinea pigs. In comparison with $L$. major and $L$. infantum promastigotes, both PSC and ASC reagents exhibited similar and in some tests higher levels of sensitivity and could be interchangeably used for in-vivo stimulations. Altogether, these results indicated the potency of L. infantum ASC or PSC to stimulate the $\mathrm{T}$ cells which upon further refinement could function as skin test reagents for VL epidemiological or vaccine studies. In this regard, PSC appear to be a better candidate, due to its less laborious preparation procedure.

\section{ACKNOWLEDGEMENT}

This project was funded by research grant \# IPI-195 from Pasteur Institute of Iran. This article has been extracted from a portion of a M.Sc. thesis written by Ali Najafi Dastanaei from Department of Immunology, Pasteur Institute of Iran.

\section{CONFLICT OF INTEREST}

The authors declare that they have no conflict of interest.

\section{REFERENCES}

1. Mougneau E, Bihl F, Glaichenhaus N. Cell biology and immunology of Leishmania. Immunol Rev. 2011;240(1):28696. doi:10.1111/j.1600-065X.2010.00983.x.

2. Magill AJ. Leishmania Species : Visceral (Kala-Azar), Cutaneous, and Mucosal Leishmaniasis in: Mandell: Mandell, Douglas, and Bennett's Principles and Practice of Infectious Diseases. 7 ed. Churchill Livingstone: Elsevier; 2010 .

3. Alvar J, Velez ID, Bern C, Herrero M, Desjeux P, Cano J et al. Leishmaniasis worldwide and global estimates of its incidence. PLoS One. 2012;7(5):e35671.

doi:10.1371/journal.pone.0035671.

4. WHO. Control of the Leishmaniases: report of ameeting of the WHO expert committee on the control ofleishmaniases, Geneva, 22-26 March 2010. WHO Techni-cal Report Series. 2010 .

5. Schonian G, Mauricio I, Cupolillo E. Is it time to revise the nomenclature of Leishmania? Trends Parasitol. 2010;26(10):466-9. doi:10.1016/j.pt.2010.06.013.

6. Chappuis F, Sundar S, Hailu A, Ghalib H, Rijal S, Peeling RW et al. Visceral leishmaniasis: what are the needs for diagnosis, treatment and control? Nat Rev Microbiol. 2007;5(11):873-82. doi:10.1038/nrmicro1748.

7. Desjeux P. Leishmaniasis: current situation and new perspectives. Comp Immunol Microbiol Infect Dis. 2004;27(5):305-18. doi:10.1016/j.cimid.2004.03.004.

8. DWT. C. First WHO Report on Neglected Tropical Diseases: Working to Overcome the Global Impact of Neglected Tropical Diseases. World Health Organization, Geneva, Switzerland, pp. 91-96. Available form: http:// www.who.int/neglected_disease/2010 report/en/. 2010.

9. Sacks D, Noben-Trauth N. The immunology of susceptibility and resistance to Leishmania major in mice. Nat Rev Immunol. 2002;2(11):845-58. doi:10.1038/nri933 nri933 [pii].

10. Mauel J, Behin R. Leishmaniasis: Immunity, immunopathology and immunodiagnosis. In: Cohen $\mathrm{S}$, 
Warren KS, eds. Immunology of Parasitic Infection. Oxford: Blackwell Scientific Publication; 1982: 312. 1982.

11. Manson-Bahr PEC. Diagnosis. In: Peters W, Killickkendrick R, Editors. The Leishmaniases inbiology and medicin, vol. 2, clinical aspects and control. london: academic Press; 1987: 703-29. 1961/11/01 ed. Trans R Soc Trop Med Hyg. Academic press; 1987.

12. Khabiri AR, Bagheri F, Alimohammadian MH, Assmar M, Nadaf SR. Leishmanin skin test in guinea pig with a single purified protein of Leishmania major. Exp Parasitol. 2005;111(4):239-43. doi:10.1016/j.exppara.2005.08.011.

13. Manson-Bahr PE. Immunity in kala-azar. Trans R Soc Trop Med Hyg. 1961;55:550-5.

14. Pampiglione S, Manson-Bahr PE, La Placa M, Borgatti MA, Musumeci S. Studies in Mediterranean leishmaniasis. 3. The leishmanin skin test in kala-azar. Trans R Soc Trop Med Hyg. 1975;69(1):60-8.

15. Weigle KA, Santrich C, Martinez F, Valderrama L, Saravia NG. Epidemiology of cutaneous leishmaniasis in Colombia: environmental and behavioral risk factors for infection, clinical manifestations, and pathogenicity. J Infect Dis. 1993;168(3):709-14.

16. Weigle KA, Santrich C, Martinez F, Valderrama L, Saravia NG. Epidemiology of cutaneous leishmaniasis in Colombia: a longitudinal study of the natural history, prevalence, and incidence of infection and clinical manifestations. J Infect Dis. 1993;168(3):699-708.

17. Souza WJ, Sabroza PC, Santos CS, de Sousa E, Henrique MF, Coutinho SG. Montenegro skin tests for American cutaneous leishmaniasis carried out on school children in Rio de Janeiro, Brazil: an indicator of transmission risk. Acta Trop. 1992;52(2-3):111-9.

18. Kar K. Serodiagnosis of leishmaniasis. Crit Rev Microbiol. 1995;21(2):123-52.

doi:10.3109/10408419509113537.

19. Bahar K, Dowlati Y, Shidani B, Alimohammadian MH, Khamesipour A, Ehsasi $\mathrm{S}$ et al. Comparative safety and immunogenicity trial of two killed Leishmania major vaccines with or without BCG in human volunteers. Clin Dermatol. 1996;14(5):489-95.

20. Alimohammadian MH, Khamesipour A, Darabi H, Firooz A, Malekzadeh S, Bahonar A et al. The role of BCG in human immune responses induced by multiple injections of autoclaved Leishmania major as a candidate vaccine against leishmaniasis. Vaccine. 2002;21(3-4):174-80.

21. Satti IN, Osman HY, Daifalla NS, Younis SA, Khalil EA, Zijlstra EE et al. Immunogenicity and safety of autoclaved Leishmania major plus BCG vaccine in healthy Sudanese volunteers. Vaccine. 2001;19(15-16):2100-6.
22. Sharifi I, FeKri AR, Aflatonian MR, Khamesipour A, Nadim A, Mousavi MR et al. Randomised vaccine trial of single dose of killed Leishmania major plus BCG against anthroponotic cutaneous leishmaniasis in Bam, Iran. Lancet. 1998;351(9115):1540-3. doi:10.1016/s0140-6736(98)09552$\mathrm{X}$.

23. Momeni AZ, Jalayer T, Emamjomeh M, Khamesipour A, Zicker F, Ghassemi RL et al. A randomised, double-blind, controlled trial of a killed L. major vaccine plus BCG against zoonotic cutaneous leishmaniasis in Iran. Vaccine. 1999;17(5):466-72.

24. Mayrink W, da Costa CA, Magalhaes PA, Melo MN, Dias $\mathrm{M}$, Lima $\mathrm{AO}$ et al. A field trial of a vaccine against American dermal leishmaniasis. Trans R Soc Trop Med Hyg. 1979;73(4):385-7.

25. Mayrink W, Williams P, da Costa CA, Magalhaes PA, Melo MN, Dias $M$ et al. An experimental vaccine against American dermal leishmaniasis: experience in the State of Espirito Santo, Brazil. Ann Trop Med Parasitol. 1985;79(3):259-69.

26. Nacy CA, Fortier AH, Pappas MG, Henry RR. Susceptibility of inbred mice to Leishmania tropica infection: correlation of susceptibility with in vitro defective macrophage microbicidal activities. Cell Immunol. 1983;77(2):298-307.

27. TDR, WHO. Leishmaniasis. In: Tropical Disease Research. Progress 1992, Eleventh pogramme Report, Geneva: UNDP/World Bank/WHO, Special Programme for Research and Training in Tropical Diseaes; 1993: 77-87.

28. Ephros M, Waldman E, Zilberstein D. Pentostam induces resistance to antimony and the preservative chlorocresol in Leishmania donovani promastigotes and axenically grown amastigotes. Antimicrob Agents Chemother.

1997;41(5):1064-8.

29. Mengeling BJ, Zilberstein D, Turco SJ. Biosynthesis of Leishmania lipophosphoglycan: solubilization and partial characterization $ר$ of the initiating mannosylphosphoryl transferase. $\neg$ Glycobiology. 1997;7(6):847-53.

30. Saar Y, Ransford A, Waldman E, Mazareb S, AminSpector S, Plumblee $\mathrm{J}$ et al. Characterization of developmentally-regulated activities in axenic amastigotes of Leishmania donovani. Mol Biochem Parasitol. 1998;95(1):920.

31. Clancy J, J. Delayed type hypersensitivity (DTH) In: Basic concept in Immunology. New York :The McGraw-Hill Companies; : 125-37. 1998.

32. Montenegro J. A cutis reacao na leishmaniose. Ann Far Med Sao Paulo ; 1:323-30. 1926. 